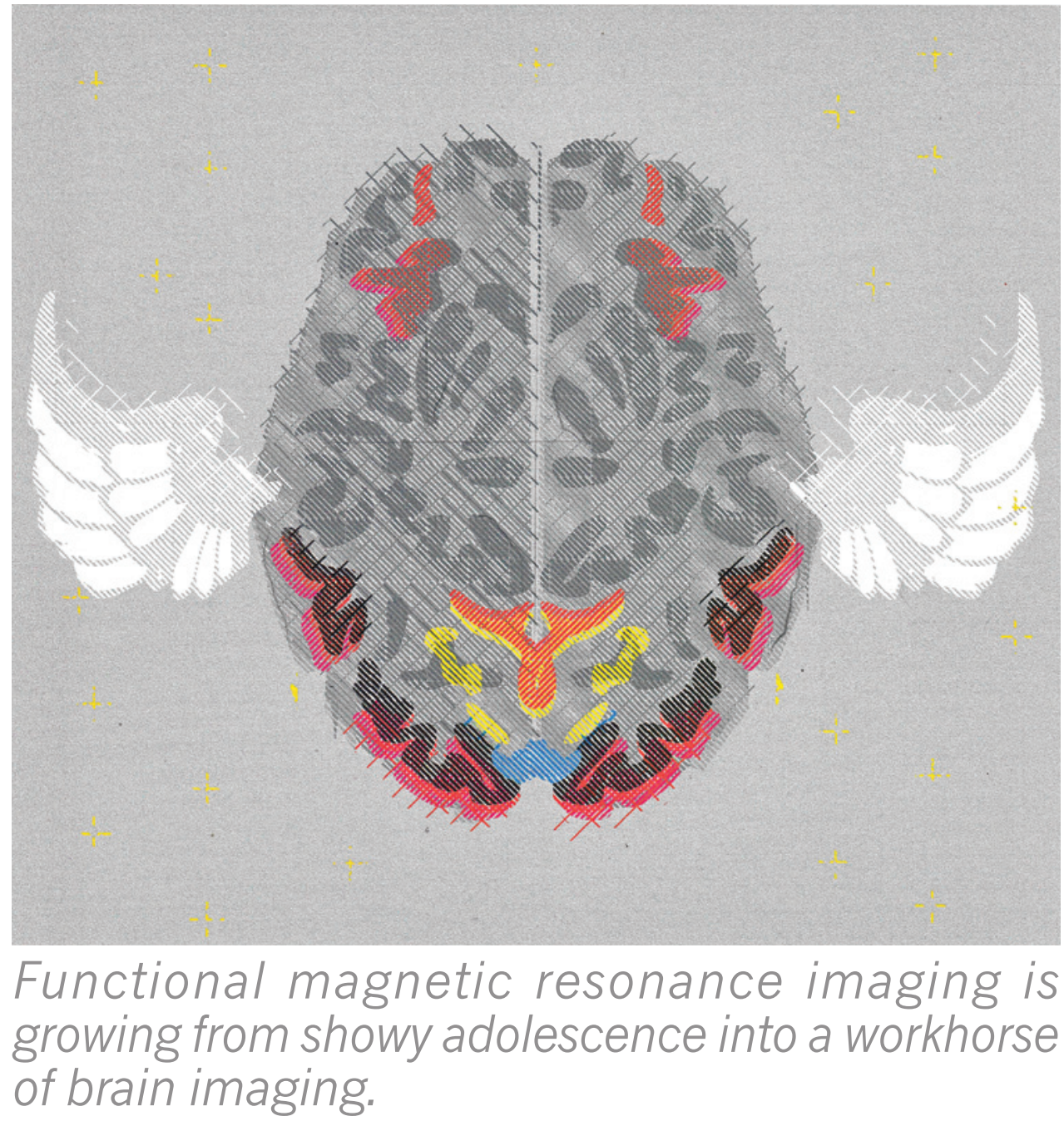

he blobs appeared 20 years ago. Two teams, one led by Seiji Ogawa at Bell Laboratories in Murray Hill, New Jersey, the other by Kenneth Kwong at Massachusetts General Hospital in Charlestown, slid a handful of volunteers into giant magnets. With their heads held still, the volunteers watched flashing lights or tensed their hands, while the research teams built the data flowing from the machines into grainy images showing parts of the brain illuminated as multicoloured blobs.

The results showed that a technique called functional magnetic resonance imaging (fMRI) could use blood as a proxy for measuring the activity of neurons - without the injection of a signal-boosting compound $^{1,2}$. It was the first demonstration of fMRI as it is commonly used today, and came just months after the technique debuted - using 
a contrast agent - in humans ${ }^{3}$. Sensitive to the distinctive magnetic properties of blood that is rich in oxygen, the method shows oxygenated blood flowing to active brain regions. Unlike scanning techniques such as electroencephalography (EEG), which detects electrical activity at the skull's surface, fMRI produces measurements from deep inside the brain. It is also non-invasive, which makes it safer and more comfortable than positron emission tomography (PET), in which radioactive compounds are injected and traced as they flow around the body.

fMRI has been applied to almost every aspect of brain science since. It has shown that the brain is highly compartmentalized, with specific regions responsible for tasks such as perceiving faces ${ }^{4}$ and weighing up moral responsibility ${ }^{5}$; that the resting brain is in fact humming with activity $^{6}$; and that it may be possible to communicate with patients in a vegetative state by monitoring their brain activity ${ }^{7}$. In 2010, neuroscientists used fMRI in more than 1,500 published articles (see 'The rise of $\mathrm{fMRI}$ ').

But researchers readily admit that the technique has flaws. It doesn't measure neuronal activity directly and it is blind to details such as how many neurons are firing, or whether firing in one region amplifies or dampens activity in neighbouring areas. The signal - a boost in blood flow in response to a stimulus - can be difficult to extract from the 'noise' of routine changes in blood flow, and the statistical techniques involved are easy to misunderstand and misuse. "I'm surprised that fMRI has kept going for 20 years," says Karl Friston, scientific director of University College London's neuroimaging centre. Friston says he thought all the interesting questions would have been "cherry-picked within the first two or three years".

But fMRI has kept going, in part because no other technique has bettered its ability to see what the human brain is doing. It has turned psychology "into a biological science", says Richard Frackowiak, who works with Friston. Now, scientists are intent on finding ways around some of the limitations and pushing the technique into the next 20 years. Nature takes a look at four futures for fMRI.

\section{DIRECT MEASURES}

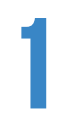

Perhaps the biggest conundrum in fMRI is what, exactly, the technique is measuring. Researchers know that it measures the oxygen carried in blood by haemoglobin, and they assume that a stronger signal reflects a greater demand for oxygenated blood when neurons become electrically active in response to a task. But several papers have called this assumption into question, suggesting that blood oxygen levels could rise in preparation for neuronal activity as well as during it ${ }^{8}$; or, worse, that they could be undulating for reasons other than neuronal activity'.

Most researchers in the fMRI community are comfortable enough with the proxy to carry on doing experiments, even if not all the details have been ironed out. "We have a pretty good handle that it's measuring something that neurons are doing that's relevant to mental function," says Russell Poldrack, director of the Imaging Research Center at the University of Texas at Austin. But some teams want to do better, by getting a more direct measure of neuronal activity. "The thing that we're most interested in is not where blood flow is but where the brain is electrically active," says John George, an MRI physicist at the Los Alamos National Laboratory in New Mexico. The only ways in which electrical activity can be measured directly, however, are by placing electrodes into the brain, or by picking up electrical signals from outside the skull, a method that lacks the depth and spatial resolution of fMRI.
One solution might be to use a type of MRI that can measure the magnetic field of each neuron as it conducts electrical signals. But these perturbations are an order of magnitude smaller than those produced by changes in blood oxygen level. George's team is therefore developing a technique that uses ultrasensitive magnetometers called SQUIDs (superconducting quantum interference devices) to pick up such perturbations ${ }^{10}$. "We detect currents close to the levels we anticipate neurons would produce," he says. But the obstacles are huge. "It's very much like the early days of fMRI," says George. The next steps are to make the detection methods faster - neural signals are much quicker than those from blood - and to win over sceptics with a clear demonstration of the measurements in a tissue sample or an animal. "There are hints that signals are there, but most people don't believe it," says George. "Once they believe you can do it, they'll show you how to do it better."

\section{MORE THAN A PRETTY PICTURE}

The multicoloured splodges that correspond to active brain areas have helped fMRI to earn the disparaging nickname 'blobology', reflecting some neuroscientists' frustration with the limited information that a blob conveys. It can show that a language task, for example, correlates with activity in the left hemisphere's frontal lobe, but not whether the activity is actually the result of language processing - or simply of paying attention to a screen. "You can't just infer causality from looking at where a task is happening," says Peter Bandettini, who heads the functional imaging methods section at the US National Institute of Mental Health's Laboratory of Brain and Cognition in Bethesda, Maryland. That is why the use of fMRI to show that a region is correlated with a task "is starting to slow down", he says. "No one's getting tenure based on that any more."

Neuroscientists are now seeking ways to build a more detailed model of the brain's organization, networks and function, so that they can interpret the patterns of activation with more confidence. A good model of brain networks might provide more detail about what happens when a person looks at a familiar face, for example, including which regions are involved in visual processing, memories and emotion; the order in which the regions respond; and how important each area is to the overall task. "The major shift is towards networks," says Stephen Smith, associate director of the Oxford University Centre for Functional MRI of the Brain, UK, whose team is working on such models. "What we're trying to get is the true underlying connectivity," he says, "rather than make a superficial comment about everything being connected to everything because they're all correlated.'

A sophisticated picture of brain networks is also the goal of the Human Connectome Project ${ }^{11}$, a 5-year, US\$40million effort funded by the US National Institutes of Health (NIH) in Bethesda, Maryland, that got under way in 2010. The project aims to map the human brain's wiring using a variety of techniques, including fMRI. Such a 'reference' connectome could help in interpreting individuals' fMRI scans and could reveal how variations in connectomes affect behaviour or contribute to disease.

Other researchers are using sophisticated statistical techniques to pick out detailed patterns from fMRI scans. One, called multivariate analysis, charts the behaviour of many units - or voxels - of brain activity in parallel, rather than averaging them together into a blob. 
Blobs can identify large, active brain areas, but might miss clumps of inactive neurons within it or small islands of active neurons in quiet areas. "The more you look, the more you get meaningful information," says Bandettini. "What previously was noise is now suddenly signal." These techniques are even allowing researchers to work out what stimuli are present just by looking at brain activity patterns. Last year, Jack Gallant from the University of California, Berkeley, recorded the fMRI activity of three members of his lab as they watched hours of film clips. The team then developed a computational model that used fMRI scans to reconstruct a movie approximating what the people had been watching - a person wearing blue, for example, or a red bird ${ }^{12}$.

\section{DAMPENING THE NOISE}

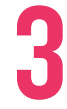

fMRI tends to generate small signals and a lot of noise. "You need quite a lot of neurons firing in synchrony with each other to see a change in blood oxygenation," says Smith. The noise means that many changes - a small group of neurons firing together, or subtle or quick variations in oxygenated blood flow - might not be picked up. The low signal-to-noise ratio forces fMRI researchers to use statistical approaches to pick out what is significant in their scans - and that means that there are numerous ways to interpret a data set. "If you try them all, you're going to find something," says Poldrack.

Some groups are managing to boost the signal by using stronger magnets. In an MRI machine, a high magnetic field aligns the spins of the protons in hydrogen atoms; then radio waves knock the spins out of alignment. As the spins gradually realign, they send out a signal - or resonate - and those in areas of oxygenated blood resonate at a different frequency from those in deoxygenated blood. But only a tiny proportion of the protons react to the field and radio waves. Stronger magnets line up a greater proportion of the proton spins, which then generate a stronger signal as they realign.

The scanners used in neuroscience today typically have magnet strengths of 3 tesla, which is many thousand times stronger than Earth's magnetic field, and have a resolution of 3 cubic millimetres. But stronger magnets are creeping into practice. In 2010, for example, scientists at the University of Nottingham, UK, used a 7-tesla magnet to build a map of the human somatosensory cortex ${ }^{13}$ - which is responsible for processing touch and some aspects of movement - at a resolution of 1 cubic millimetre. The NeuroSpin facility near Paris is building an 11.7-tesla whole-body system, the strongest yet for human studies. Magnets much stronger than this cannot be used on humans, because they increase artefacts in the images and can trigger dizziness and other side effects.

Another way to increase the signal is to inject molecules that are easier to detect than oxygenated blood, in a method more akin to PET. Gary Green, director of the York Neuroimaging Centre at the University of York, UK, is working with parahydrogen, a 'hyperpolarized' molecule in which the proton spins are more aligned than in many other molecules, and which generates a strong signal during MRI. In 2009, Green and his colleagues showed that they could transfer spins from parahydrogen to an organic molecule without changing the latter's chemical structure ${ }^{14}$ - the first step towards preparing hyper-

$\rightarrow$ NATURE.COM Listen to a podcast about 20 years of fMRl:

go.nature.com/ufbguk polarized drugs or other molecules that bind to receptors, and then track how these substances are taken up, or how they interact.

Finding better statistical ways to remove noise will also be a big help. Poldrack runs a 'best practice' wiki (www.fmrimethods.org) that covers how
fMRI data should be analysed, and has published guidelines for how the work should be reported, recommending, for example, that researchers include all the experimental detail necessary to reproduce an analysis, such as "what your subjects were asked to do and what they actually did"15. "We need to enforce more rigour," he says.

\section{WHICH WAY TO THE CLINIC?}

Getting fMRI to the clinic is, for some, the most pressing challenge the field will face in the next few years. "It hasn't really been used clinically yet, on individual subjects," says Bandettini. Clinicians want to be able to ask, for example, whether a drug is working to relieve schizophrenia, or whether a person with depression is in danger of committing suicide. The difficulty lies in making sense of an individual's scan. Most fMRI data are averages of results from many people doing the same task. This method has a higher chance of seeing a true difference between two groups or two tasks than those from an individual.

Researchers are now developing statistical methods to pull meaningful information out of a single scan. In one study ${ }^{16}$ published in 2010, a team trained a computer to pick out patterns in brain-scan data collected when participants were resting. They did this for nearly 240 people aged 7-30 years to build up maps of brain connectivity at different ages. They then showed that they could take a single brain scan from a different person and, by comparing it with their reference set, work out the owner's brain maturity. Such techniques might eventually be used to diagnose a developmental delay or psychiatric disorder, and there are hints that they can identify teenagers genetically at risk for depression ${ }^{17}$.

Having a good reference set will form the backbone of clinical fMRI, says Arthur Toga, a neurologist at the University of California, Los Angeles. Toga is a principal investigator on an effort to build such a reference, called the Alzheimer's Disease Neuroimaging Initiative, a longitudinal study of around 800 people looking at the onset and progression of Alzheimer's disease through genetic analyses, brain structure and function and blood biomarkers. Toga hopes that the information will form a database against which future individual scans can be compared.

With new ways both to examine the data and to boost the technology, many neuroscientists see a future filled with multicoloured blobs - albeit sharper and better-understood ones. "People will be very busy easily for the next 20 years," says Bandettini. "I would say that fMRI in many aspects hasn't really even begun."

Kerri Smith is editor of the Nature Podcast, and is based in London.

1. Ogawa, S. et al. Proc. Natl Acad. Sci. USA 89, 5951-5955 (1992).

2. Kwong, K. K. et al. Proc. Natl Acad. Sci. USA 89, 5675-5679 (1992).

3. Belliveau, J. W. et al. Science 254, 716-719 (1991).

4. Kanwisher, N., McDermott, J. \& Chun, M. M. J. Neurosci. 17, 4302-4311 (1997)

5. Greene, J. D., Sommerville, R. B., Nystrom, L. E., Darley, J. M. \& Cohen, J. D. Science 293, 2105-2108(2001)

6. Smith, S. M. et al. Proc. Natl Acad. Sci. USA 106, 13040-13045 (2009)

7. Monti, M. M. et al. N. Engl. J. Med. 362, 579-589 (2010).

8. Sirotin, Y. B. \& Das, A. Nature 457, 475-479 (2009).

9. Maier, A. et al. Nature Neurosci. 11, 1193-1200 (2008).

10. Matlachov, A. N., Volegov, P. L., Espy, M. A., George, J. S. \& Kraus, R. H. Jr J. Magn. Reson. 170, 1-7 (2004).

11.Bardin, J. Nature 483, 394-396 (2012)

12. Nishimoto, S. et al. Curr. Biol. 21, 1641-1646 (2011).

13.Sanchez-Panchuelo, R. M., Francis, S., Bowtell, R. \& Schluppeck, D. J. Neurophysiol. 103, 2544-2556 (2010).

14.Adams, R. W. et al. Science 323, 1708-1711 (2009).

15.Poldrack, R. A. et al. Neurolmage 40, 409-414 (2008),

16.Dosenbach, N. U. F. et al. Science 329, 1358-1361 (2010).

17. Mourão-Miranda, J. et al. PLoS ONE 7, e29482 (2012). 